\title{
KNOWN AND UNKNOWN MATTER
}

\author{
John ELLIS \\ Theoretical Physics Division, CERN \\ CH - 1211 Geneva 23
}

\begin{abstract}
Among the known forms of matter discussed in this lecture are the proton, neutron, electron and $Z$ particle. Among the less well-known forms of matter are neutrinos and the quarkgluon plasma. The neutrinos make this list because of the possibility that they may have mass and hence constitute part of the Dark Matter in the Universe. Among the unknown forms of matter discussed in this lecture are Higgs bosons and supersymmetric particles. The former, in its Grand Unified guise, may have been responsible for inflation, while the lightest supersymmetric particle is one of the best candidates for Cold Dark Matter.
\end{abstract}

Talk given at the EPS General Meeting on Physics

"Trends in Physics"

Florence, September 1993

CERN-TH.7125/93

December 1993 


\section{The Big Bang and cosmological nucleosynthesis}

According to the Big Bang model, the origin of the Universe was a (sort of) simultaneous explosion everywhere in space, after which the remnants separated and cooled in such a way that

$$
a \cdot T \simeq \text { constant }
$$

where $a$ is the scale size of the Universe and $T$ is its temperature. Historically, there have been two important pieces of evidence for the Big Bang, in addition to the observed present-day Hubble expansion.

One is the fact that we are based in a microwave background radiation with an effective temperature of $2.7 \mathrm{~K}$. According to the Big Bang model, this radiation was emitted when the Universe was about 1,000 times hotter and smaller than today, at the epoch when ions and electrons combined to form neutral atoms. The presence of this microwave background radiation means that we leave in a gas of photons $\gamma$ with a number density

$$
n_{\gamma} \simeq 400 \mathrm{~cm}^{-3}
$$

and we expect the Universe to contain a similar density of neutrinos.

The second important piece of evidence for the Big Bang model is provided by the abundances of the light nuclei. For example, all stars, galaxies, etc., which we can measure contain at least $24 \%$ by mass of ${ }^{4} \mathrm{He}$. It is believed that this and the smaller abundances of other light elements $\left(\mathrm{D},{ }^{3} \mathrm{He},{ }^{7} \mathrm{Li}\right)$ were cooked by the Universe when it was about $10^{9}$ times hotter and smaller than it is today. The temperature of the Universe would have been about $10^{9} \mathrm{~K}$ when its age was about 100 secs. During the early history of the Universe, when its expansion was dominated by relativistic matter, the age $t$ would have been related to the temperature $T$ by the following approximate relation

$$
t(\mathrm{sec}) \simeq[T(\mathrm{MeV})]^{-2}
$$

with the numerical coefficient depending on the number of particle species. As seen in Fig. 1, the consistency with observation of calculations of nucleosynthesis in a homogeneous Big Bang requires there to be only a small number of neutrino species and the baryon density $\rho_{B}$ today to be less than the critical density $[1] \rho_{C}$ :

$$
\Omega_{B} \equiv \rho_{B} / \rho_{C} \sim 0.01 \text { to } 0.1
$$

which is rather more than that seen shining in stars, etc, ....

Going back in time, the next interesting event in the history of the Universe which we encounter is the transition from quarks and gluons to protons and neutrons. Protons and neutrons are known to be extended objects, about $10^{-13} \mathrm{~cm}$ across, which are made out of confined quarks. Nuclei containing many protons and neutrons contain three times as many quarks, but under normal circumstances these are confined in separate bags for all the individual nucleons. However, just as atoms ionize at high temperature (cf. the microwave background 
radiation), we expect quarks to "ionize" or deconfine at high temperatures, above about 200 $\mathrm{MeV}$ or $2 \times 10^{12} \mathrm{~K}$. Above this temperature, hadronic matter is believed to exist in the form of a plasma of quarks and gluons. As the Universe expanded and cooled, the quarks and gluons would have combined to form protons, neutrons and other strongly-interacting particles, perhaps leading also to inhomogeneities in the early Universe.

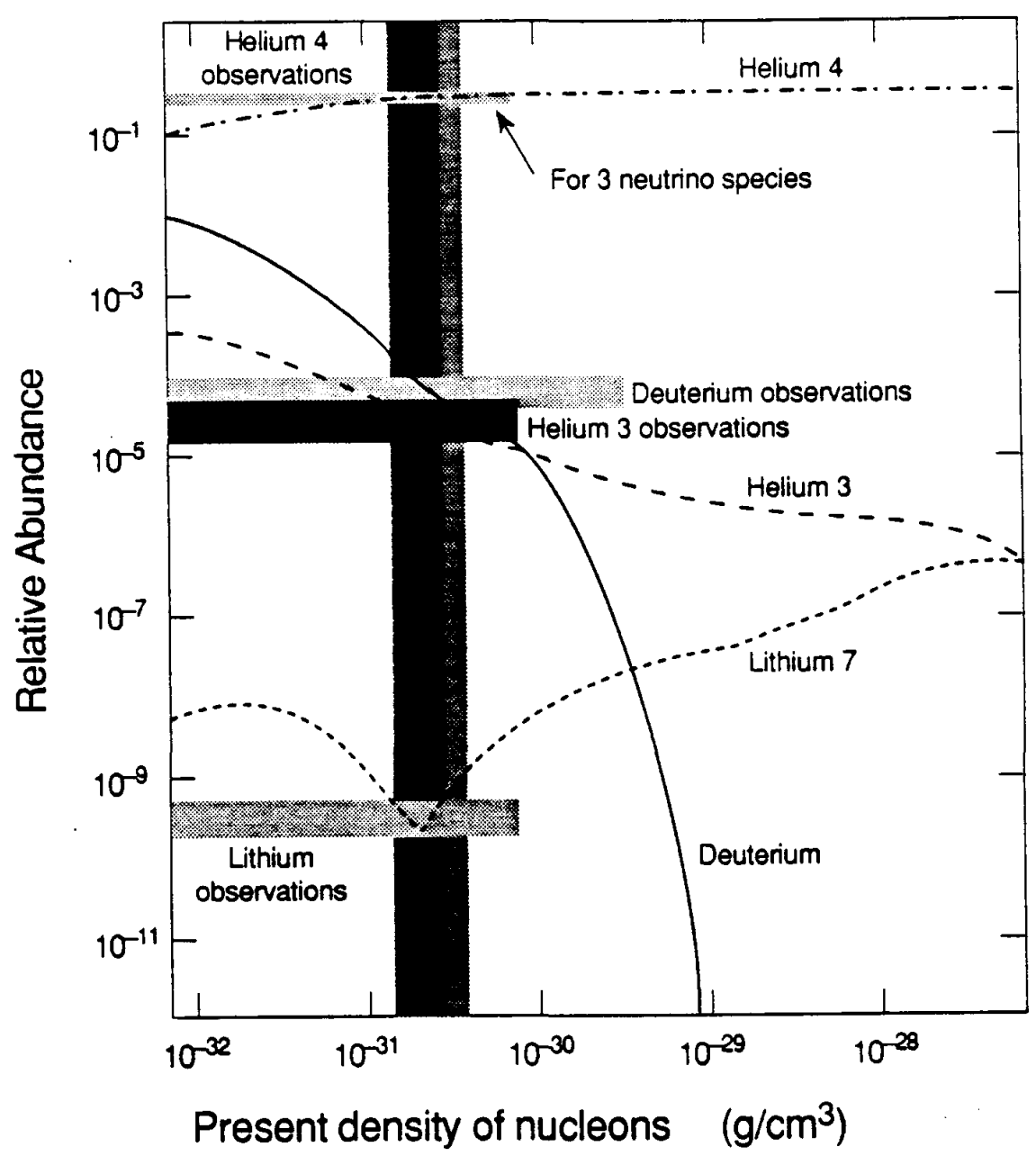

Fig. 1 - Abundances of the light elements: confrontation between data and predictions based on homogeneous Big Bang nucleosynthesis [1].

It has been suggested [2] such these inhomogeneities might relax the above upper bound on the density of strongly-interacting matter in the Universe today, and the possibility has even been raised that the baryons could provide the critical density and all the dark matter beloved of astrophysicists. This possibility seems to be disfavoured by recent theoretical estimates of the parameters of the quark-hadron phase transition [3]. The existence of the quark-gluon 
plasma and the nature of the phase transition are being explored by experimental programmes colliding heavy nuclei at Brookhaven and at CERN [4].

Inhomogeneities could have arisen during the quark-gluon phase transition, and possibly affected Big Bang nucleosynthesis if the transition was first-order. In a first-order transition, bubbles of the new vacuum form separated by regions of the old phase. If the distance between nucleation sites of the new phase is larger than the characteristic diffusion length of protons in the early Universe

$$
d \gtrsim 0.5 \mathrm{~m}
$$

then neutrons diffuse more than protons, and the $n / p$ ratio as well as the $\eta=n_{B} / n_{\gamma}$ could vary through space. This could lead to different light element abundances, and hence possibly a relaxation of the cosmological upper bound on the present baryon density [2].

However, calculations of this scenario suggest that if $\Omega_{B}=1$ then there is an overabundance of ${ }^{7} \mathrm{Li}$. This problem could be avoided only if there was some special process able to deplete the cosmological ${ }^{7} \mathrm{Li}$ abundance, but none is known. Recent studies [5] indicate that there is also a problem with the abundance of ${ }^{4} \mathrm{He}$, which is also overproduced, and with deuterium $D$, which tends to be underproduced in such an inhomogeneous nucleosynthesis scenario. As seen in Fig. 2, the general conclusion is that $\Omega_{B}=1$ is excluded, that there is very little room for any substantial deviation from the standard homogeneous Big Bang nucleosynthesis scenario, and hence that

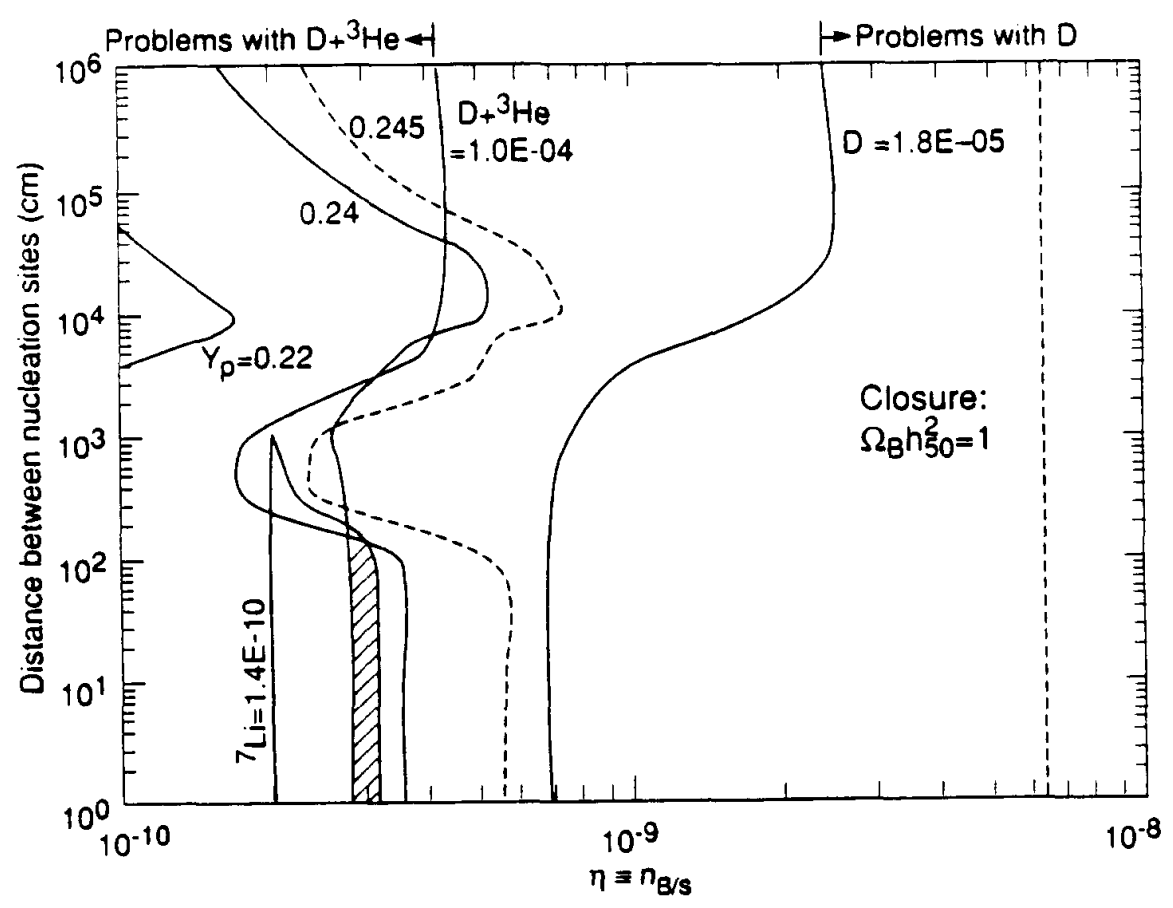

Fig. 2 - Constraints on inhomogeneous Big Bang nucleosynthesis models imposed by the observed light element abundances [5].

$$
n_{B} / n_{\gamma} \simeq 3 \times 10^{-10}
$$


It seems that a strong first-order quark-hadron transition is disfavoured by astrophysical as well as theoretical considerations.

\section{Aspects of the Standard Model}

According to the Standard Model [6], electromagnetic interactions are carried by photons, which have mass zero and an infinite effective range. On the other hand, the weak interactions responsible for radioactivity are carried by $W^{ \pm}$and $Z^{0}$ particles weighing about $80 \mathrm{GeV}$ and $91.187 \mathrm{GeV}$ respectively, with an effective range of about $10^{-16} \mathrm{cms}$. The biggest puzzle in the Standard Model is why the apparently similar $\gamma, W^{ \pm}$and $Z^{0}$ have such different masses. Table 1 exhibits the elementary particles of matter, namely the quarks that make up stronglyinteracting matter and the weakly-interacting leptons. The masses of these elementary matter particles are also indicated. It should be recalled that the top quark $t$ has not yet been discovered, and the mass $m_{t} \sim 150 \mathrm{GeV}$ is estimated from the analysis of precision electroweak data from LEP and elsewhere [7]. It should also be noted that we only have upper limits on the neutrino masses, and that the possible existence of known-zero neutrino masses will be an important theme later in this talk. It is LEP that has told us that there can only be three species of light neutrinos. By determining the rate of $Z^{0}$ decays into invisible neutral particles, experimentalists at LEP have measured an effective number of neutrino species [6]

$$
N_{\nu}=2.982 \pm 0.027
$$

In general, experiments at LEP have tested and verified the Standard Model with a precision between $1 \%$ and $0.1 \%$. These experiments recreate conditions when the temperature of the Early Universe was about $100 \mathrm{GeV} \sim 10^{15} \mathrm{~K}$, which was reached when the age of the Universe $t \sim 10^{-10}$ secs.

Table 1: Matter particles in the Standard Model

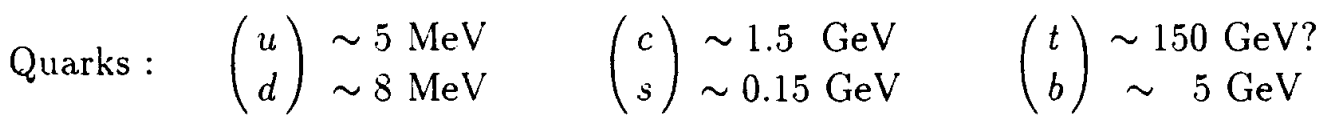

$$
\begin{aligned}
& \text { Leptons: }\left(\begin{array}{c}
\nu_{e} \\
e
\end{array}\right) \begin{array}{c}
<7 \mathrm{eV} \\
\frac{1}{2} \mathrm{MeV}
\end{array} \quad\left(\begin{array}{c}
\nu_{\mu} \\
\mu
\end{array}\right) \begin{array}{c}
<0.27 \mathrm{MeV} \\
0.1 \mathrm{GeV}
\end{array} \quad\left(\begin{array}{c}
\nu_{\tau} \\
\tau
\end{array}\right) \begin{array}{r}
<31 \mathrm{MeV} \\
1.38 \mathrm{GeV}
\end{array}
\end{aligned}
$$

As already mentioned, one of the biggest puzzles of the Standard Model is the origin of mass [8]. The fact that the $W^{ \pm}$and $Z^{0}$ masses are non-zero and different from that of the $\gamma$ tells us that the underlying electroweak symmetry must be broken. In the Standard Model, this symmetry breaking is due to a coupling to a ubiquitous scalar "Higgs" field. The mechanism of mass generation and symmetry breaking is much like that in the BCS theory of superconductivity. In that theory, there is an effective photon mass in a superconductor, due to the coupling of the $\gamma$ to an effective scalar field made out Cooper pairs of electrons $(e e)$. The 
symmetry breaking in the Standard Model would correspond to a very high $T_{c}$ superconductor indeed! The electroweak symmetry is believed to be restored above $T \sim 100 \mathrm{GeV} \sim 10^{16} \mathrm{~K}$, a temperature which would have been reached when the Universe was $\sim 10^{-12}$ secs. old.

By analogy with the Cooper pairs of conventional superconductivity, it has been suggested that the Higgs field of the Standard Model may be composite, but theories with composite Higgs bosons seem difficult to reconcile with the precision data available from LEP [7]. An elementary Higgs particle is being sought actively at LEP, and will be one of the main goals of research at the LHC. In order to avoid large quantum corrections to the mass of an elementary Higgs boson and hence to the $W^{ \pm}$and $Z^{0}$ bosons, it is believed likely that an elementary Higgs boson must be accompanied by other particles, as in a supersymmetric theory [9]. In such a theory, all the known particles would be accompanied by similar particles, with identical internal quantum numbers such as electric charge $Q$, baryon number $B$ and lepton number $L$ but with spins differing by half a unit. In this way, a supersymmetric theory contains equal numbers of bosons and fermions with identical couplings, whose virtual quantum corrections to the mass of the Higgs boson cancel. For reasons discussed in more detail later, the lightest supersymmetric particle is a good candidate for most of the dark matter in the Universe [10].

\section{GUTs and Inflation}

One of the other outstanding problems left unsolved by the Standard Model is that of the grand unification of the strong and electroweak interactions. LEP data are consistent [11] with the simplest supersymmetric Grand Unified Theories (GUTs) with unification at a temperature scale $T \sim(1$ or 2$) \times 10^{16} \mathrm{GeV} \sim 10^{29} \mathrm{~K}$, which would have been reached when the Universe was about $10^{-36}$ secs. old.

One of the most active and interesting areas at the interface between particle physics and cosmology nowadays is inflation [12], which could well have taken place around this GUT scale, driven by a GUT analogue of the Higgs field discussed in the previous section. Data from COBE [13] and other experiments may be providing us for the first time with direct observations of the primordial perturbations that have led to the formation of structure in the Universe. Motivations [12] for inflationary cosmology are provided by various puzzles of the standard Big Bang cosmology. One is the horizon/smoothness problem, which is that distant parts of the Universe that are just now becoming visible, i.e., coming within each other's apparent horizons, are "similar". The best evidence for this is provided by the isotropy of the cosmic microwave background radiation

$$
\left(\frac{\delta T}{T}\right)_{C M B R} \lesssim 10^{-5}
$$

but also by the increasing homogeneity of the matter distribution in the Universe at large distances. This smoothness is a puzzle, because previously these distant parts of the Universe would have been unable to communicate in standard Big Bang cosmology, since the scale size

$$
a \sim t^{\frac{1}{2}} \text { or } t^{\frac{2}{3}}
$$


for relativistic or non-relativistic matter, whereas the horizon size increases linearly with time. Another puzzle is the flatness/oldness problem. Consider the standard equation of FriedmannRobertson-Walker cosmology

$$
\left(\frac{\dot{a}}{a}\right)^{2}=H^{2}=\frac{8 \pi G_{N} \rho}{3}-\frac{k}{a^{2}}
$$

and define

$$
\Omega \equiv \frac{\rho}{\rho_{c}} \quad: \quad H^{2}=\frac{8 \pi G_{N}}{3} \rho_{c}
$$

It is easy to see from Eq. (10) that

$$
\Omega(t)=\frac{1}{\left(1-\frac{k / a^{2}}{8 \pi G_{N} \rho / 3}\right)}
$$

where $\rho \approx a^{-4}$ for a radiation-dominated Universe, and $\rho \approx a^{-3}$ for a matter-dominated Universe. This means that in order for $\Omega=O(1)$ today, one would have required

$$
|\Omega-1| \lesssim 10^{-60} \text { at } t \sim 10^{-43} s
$$

which represents a tremendous amount of fine tuning.

The basic idea of inflation [12] is that at some early epoch the energy density may have been dominated by an approximately constant term, leading, as one can see from Eq. (10), to an epoch of approximately exponential expansion

$$
a=a_{i} \exp (H t): \quad H=\sqrt{\frac{8 \pi G_{N}}{3} V}
$$

This would have caused the event horizon to expand exponentially, as seen in Fig. 3, so that the entire observable Universe could have been within a single pre-inflationary horizon volume. The homogeneity of the observed Universe could have been established at this epoch, if the exponential expansion included at least $60 e$-foldings. This inflationary epoch would also have made the Universe appear essentially flat, since the curvature term $k / a^{2}$ in Eq. (10) would have become negligible during the inflationary expansion. However, as we shall see shortly, quantum perturbations during this epoch cause $\Omega$ to differ from unity by a small amount

$$
|\Omega-1| \sim 10^{-5}
$$

The large entropy of the observed Universe would have been produced at the end of inflation, when the approximately constant energy density driving inflation was converted into matter and radiation, and the exponential expansion of the Universe would have driven away any unwanted particles produced earlier, such as monopoles or gravitinos.

The first inflationary model, old inflation [12], postulated that the exponential expansion was driven by the field energy in a false vacuum, prior to a first-order phase transition due to tunnelling through a potential barrier. This would have led to an effective cosmological 
constant while the Universe was stuck in the false vacuum with potential energy $V$, and a constant Hubble expansion rate

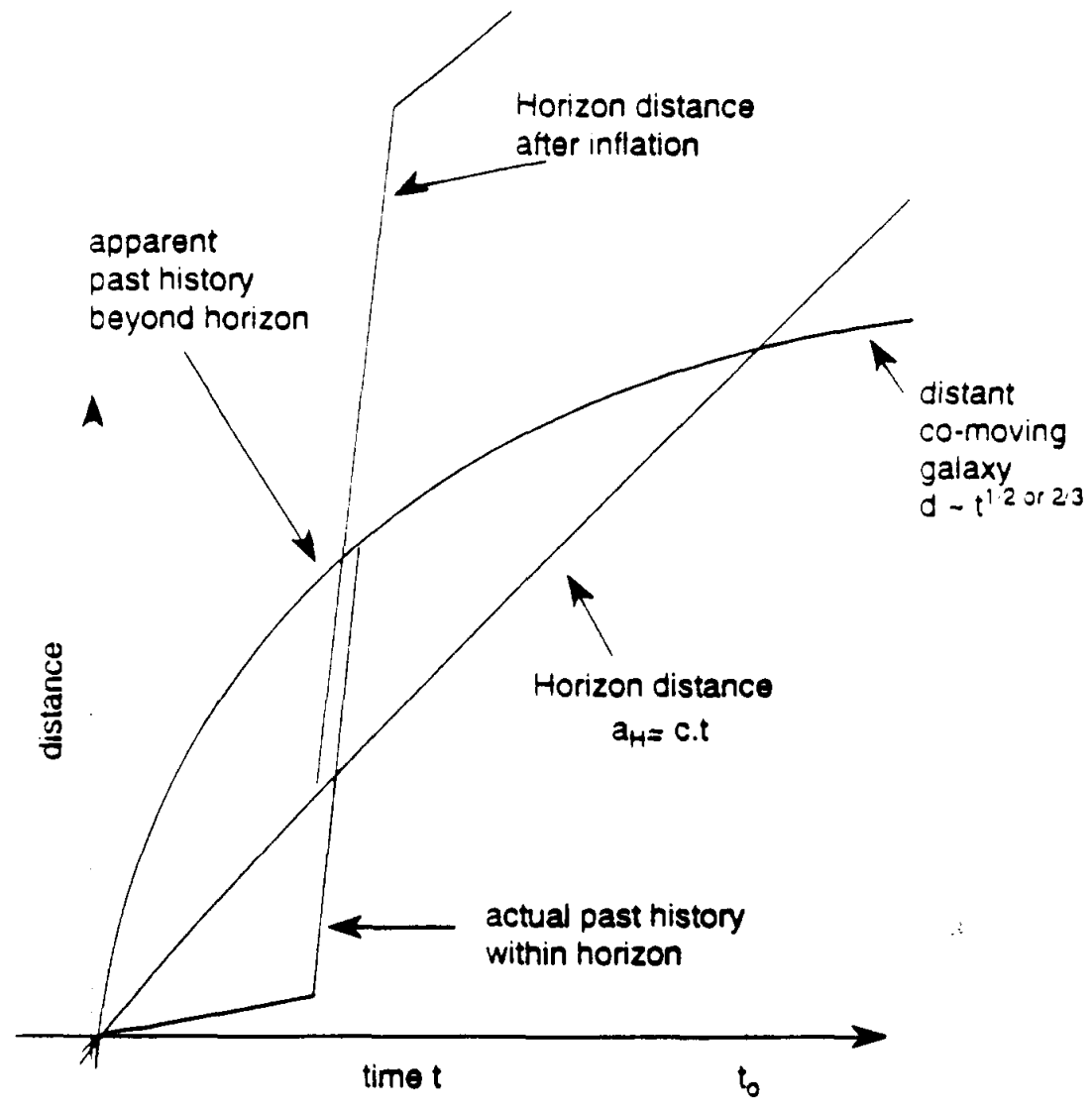

Fig. 3 - The horizon and the history of a distant galaxy at rest in a comoving reference frame, in conventional Big Bang cosmology and in inflationary cosmology.

$$
\Omega=8 \pi G_{N} V, H=\sqrt{\frac{8 \pi G_{N}}{3} V}
$$

First-order phase transitions proceed via bubble nucleation, and the problem with old inflation is that it is difficult to see [14] how the transition could ever have been completed. The basic problem is that the regions of old vacuum that surround the bubbles of true vacuum expand exponentially

$$
V_{\text {old }}(t) \sim a^{3}(t) \sim e^{3 H t}
$$

and the co-moving bubble size remains constant. Thus, for a tunnelling rate $\Gamma$, the probability of staying in the false vacuum is

$$
P_{\text {old }}(t) \sim \exp \left(\frac{-4 \pi}{3}\left(\frac{\Gamma}{H^{4}}\right) H t\right)
$$


and the fraction of the Universe in the old vacuum is

$$
F_{\text {old }}(t)=P_{\text {old }}(t) a^{3}(t) \sim \exp \left(\frac{-4 \pi}{3}\left(\frac{\Gamma}{H^{4}}\right) H t\right) \exp (3 H t)
$$

This fraction goes to zero only if $\Gamma / H^{4}>>1$, but in this case the first-order phase transition is very rapid, and there is not enough inflation.

One of the possible fixes for this problem is provided by new inflation [15], according to which the (approximately) exponential expansion occurred during the slow roll-over into the true vacuum, possibly but not necessarily after a first-order transition. This scenario requires a very flat potential in order to obtain enough $e$-foldings $H \tau \gtrsim 60$ during a very slow roll-over. According to this scenario, we live inside a bubble that expanded (approximately) exponentially, that is probably much larger than the observed Universe. It is clear that in this scenario the field energy driving inflation is not quite constant during the roll-over, and hence the Hubble expansion rate $H$ is also not quite constant [16].

According to the inflationary scenaria discussed in the previous section, perturbations arise [17] in the early Universe as a result of quantum fluctuations in scalar fields. These cause different parts of the Universe to expand differently, causing them to expand at different rates and have different densities. These quantum fluctuations yield a Gaussian random field of perturbations $\delta \rho / \rho$. which are of similar magnitudes at different scale sizes, as wanted by astrophysicists. The magnitude of the perturbations is related to the field energy

$$
\frac{\delta \rho}{\rho} \times G i \sqrt{V}
$$

The COBE data [13] that inclicate $\delta T / T \sim 10^{-5}$ correspond to an energy scale for the inflationary field

$$
V^{1 / 4} \sim 10^{16} \mathrm{GeV}
$$

which is very similar to the GUT energy scale favoured by particle theories [11]. In view of the extreme importance of the COBE data [13], it is encouraging that they have already been corroborated by data from many other experiments using different techniques, e.g., groundbased detectors and balloons operating at different frequencies.

\section{Particle Candidates for Dark Matter}

Inflation tells us to expect that the density of the Universe as a whole is indistinguishable from the critical density: $\Omega=1$. However, observations only provide $\Omega_{\text {visible }} \lesssim 0.01$, whereas Big Bang nucleosynthesis apparently restricts $\Omega_{B} \lesssim 0.1$. Measurements of rotation curves suggest that galactic haloes contain $\Omega_{\text {halo }} \sim 0.1$. Thus the halo could in principle be made out of baryons, although arguments have been given that these could not be in the form of gas, dust or snowballs [18]. However, the possibility that they could be "Jupiters" or brown dwarfs, i.e., small, "failed" stars remains open. Indeed, one of the high points of this meeting has been the announcement [19] of possible candidates for microlensing events caused by brown dwarfs in 
the galactic halo. It remains to be seen whether these account for all the dark matter in the halo of our galaxy, which has a local clensity estimated to be

$$
\rho_{\text {halo }} \sim 0.3 \mathrm{GeV} \mathrm{cm}^{-3} \sim 0.01 \mathrm{~m}_{0} p c^{-3}
$$

or whether there is a substantial contribution to the local halo density from non-baryonic dark matter.

Theorists of structure formation in the early Universe distinguish two categories of dark matter: hot, which was relativistic when galaxy-sized perturbations came within the event horizon and began to grow, and cold, which was non-relativistic at that epoch. The one you favour depends on your pet theory of galaxy formation. If you believe these formed from a Gaussian random field of perturbations laid down during inflation, as discussed in the previous sections, then cold dark matter has usually been preferred. This is because it enables perturbations to grow on all scales from galaxies upwards, whereas hot dark matter escapes from small galaxy-sized perturbations, retarding their growth. Thus, in a pure hot dark matter scenario, galaxies only form late as a result of larger structures breaking up. This scenario seemed to be disfavoured by the observation of galaxies and quasars at high redshifts, as well as by other considerations. However. if galaxies formed from seeds, such as cosmic strings, hot dark matter might be preferred.

Assuming some type of inflationary scenario as discussed earlier, the comparison of COBE and other large-scale data with other data on structures at smaller scales seems to require a modification of the pure cold dark matter scenario [20]. One possibility is that there is a non-zero cosmological constant, but this seems very unlikely from the point of view of particle physics, since there is no known natural reason why the cosmological constant should be in the range of cosmological utility. The two prime candidates for modifying the standard cold dark matter scenario seem to be a mixed dark matter with an admixture of hot dark matter

$$
\Omega_{\text {Cold }} \sim 0.7, \Omega_{\mathrm{Hot}} \sim 0.3 . \Omega_{\text {Baryons }} \lesssim 0.1
$$

or a tilt in the spectrum of primordial density perturbations. The parameters of these two models can be fixed by galaxies and by the COBE data, which fix the overall normalization of $\delta \rho / \rho$ : the question is then what the models predict at intermediate scales, in particular for the peculiar motions of clusters. At least some astrophysicists [21] will tell you that the cold dark matter with tilt model predicts peculiar motions on the scale of clusters that are too small by comparison with the data. Moreover, at least in simple models one expects very small amount of tilt. Therefore, my own preference at the moment is for a mixed dark matter model as in Eq. (23).

The relic energy density in neutrinos can be in the range of interest to astrophysicists and cosmologists for three regions of the neutrino mass, as seen in Fig. 4. When the neutrino mass is much less than $1 \mathrm{MeV}$. its number density is independent of its mass, so its energy density increases linearly with the mass and, because of their very large abundance similar to that of photons (2), becomes comparalole to the critical density when the neutrino weighs about $30 \mathrm{eV}$. A neutrino in this mass range would constitute hot dark matter. For larger neutrino masses, the neutrino density $\Omega_{\nu}$ is too large, until the neutrino mass increases to a 
few $\mathrm{GeV}$. In this region, the neutrino number density becomes suppressed by a Boltzmann factor $\exp -m_{\nu} / T$, and the relic massive neutrino density can be again of astrophysical and cosmological interest [22]. Neutrinos and many other neutral particles can annihilate through the $Z^{0}$, so their annihilation cross-sections are maximal when $m_{\nu}=m_{Z} / 2$, resulting in a local minimum of the neutrino energy density. As the neutrino mass is further increased, the annihilation cross-section decreases, resulting in an increase in the number density and hence the energy density. There is thus a second region of large neutrino masses around $1 \mathrm{TeV}$ where the neutrino could constitute cold dark matter [23]. Similar features are exhibited by the relic density of other neutral non-baryonic candidates for dark matter.

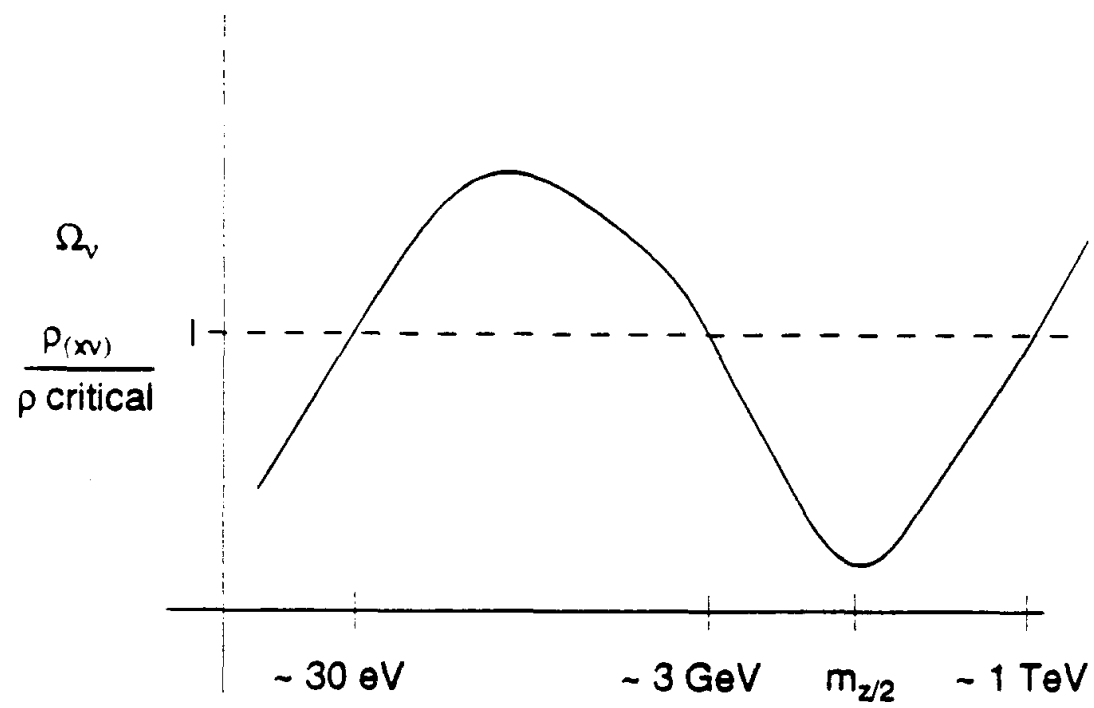

Fig. 4 - Qualitative picture of the neutrino relic density $\rho_{\nu}$ as a function of the neutrino mass $m_{\nu}$.

Data on solar neutrinos [24] may provide extra motivation for the possibility that one of the neutrinos, most probably the $\nu_{\tau}$, contributes significantly to dark matter. As is well known, all four experiments searching for solar neutrinos report a deficit compared with the standard solar model. This could be due to astrophysical reasons, but there is a very elegant explanation in terms of matter-enhanced neutrino oscillations. The preferred fit to the combined data on solar neutrinos requires

$$
\delta m_{\nu}^{2} \sim 10^{-5} \mathrm{eV}^{2}, \sin ^{2} 2 \theta \sim 10^{-2}
$$

Very light neutrino masses such as these find a natural explanation within GUTs, via the seesaw mass matrix mechanism [25]. According to this, there is mixing between the light left-handed neutrinos of the Standard Model and massive right-handed singlet neutrinos, via a mass matrix of the form

$$
\left(\nu_{L}, \bar{\nu}_{R}\right)\left(\begin{array}{cr}
\sim 0 & m_{q} \\
m_{q} & M_{\mathrm{GUT}}
\end{array}\right)\left(\begin{array}{c}
\nu_{L} \\
\overline{\nu_{R}}
\end{array}\right)
$$


where $M_{\mathrm{GUT}}$ should be within a few orders of magnitude of the grand unification mass scale $m_{X} \sim 10^{16} \mathrm{GeV}$. This mass matrix suggests the hierarchy

$$
m_{\nu_{e}}: m_{\nu_{\mu}}: m_{\nu_{e}} \simeq m_{u}^{2}: m_{\sigma}^{2}: m_{t}^{2}
$$

which, when combined with Eq. (2t). suggests that

$$
m_{\psi_{\mu}}<<m_{\nu_{\mu}} \sim 3 \times 10^{-3} \mathrm{eV}
$$

Scaling up the $\nu_{\mu}$ mass by $m_{t}^{2} / m_{c}^{2}$, we arrive at the estimate

$$
m_{\nu_{e}} \sim 10 \mathrm{eV}
$$

offering the possibility [26] that the $\nu_{\tau}$ could constitute the hot dark matter discussed in the previous section.

More detailed modelling of neutrino mixing angles finds that the value of $\sin ^{2} 2 \theta$ in Eq. (24) is very natural, and suggests that $\sin ^{2} \theta_{\mu r}$ could be in the range detectable in a new round of accelerator experiments. Two such experiments. NOMAD and CHORUS, are taking test data at CERN before the end of this year [ $2 \pi]$, and may be able to tell us within a couple of years whether the $\nu_{\tau}$ has a mass in the range of Eq. (2S) and a mixing angle $\sin ^{2} 2 \theta_{\mu \tau} \geq 10^{-4}$, as seen in Fig. 5. In the longer term, ideas are being discussed [28] at CERN for sending a neutrino beam to the Gran Sasso laboratory, a clistance of $732 \mathrm{~km}$, passing almost beneath Florence! The neutrino beam would be generated along a beam transfer line from the SPS to the LHC, and would provide a known fux of neutrinos that could be used to test suggestions of oscillations in atmospheric neutrinos.

Most supersymmetric theories contain one stable particle, which should be around today as a cosmological relic from the Big Bang [10]. This is because most supersymmetric theories have a multiplicatively-conserved quantum number called $R$ parity, that takes the value +1 for all particles and -1 for all sparticles. Its conservation is linked to $B$ and $L$ conservation, since $R$ parity may be represented as

$$
R=(-1)^{3 B+L+2 S}
$$

This expression also shows how $R$ parity may be violated, for example, by a violation of $L$ either in the vacuum through a vacuum expectation value for some sneutrino field $\dot{\nu}$, or explicitly, for example by a coupling between a Higgs field and a lepton field. However, these possibilities are severely constrained by laboratory limits on lepton-number-violating interactions, as well as by cosmological considerations. There are three important implications of $R$ parity conservation. (1) Sparticles must be produced in pairs, for example $\epsilon^{+} \epsilon^{-} \rightarrow \bar{\mu}^{+} \dot{\mu}^{-}$or $\bar{p} p \rightarrow \dot{q} \bar{g} X^{-}$. (2) Heavier sparticles must decay into lighter sparticles, for example $\dot{\epsilon} \rightarrow \epsilon \dot{\gamma}, \dot{q} \rightarrow q \dot{y}$. (3) The lightest supersymmetric particle must be stable, because it has no available decay mode.

If the stable relic particle had electromagnetic charge or strong interactions, then it would have presumably condensed along with ordinary matter into galaxies, stars and planets, and hence be detectable as an anomalous heavy isotope, with a calculated abundance

$$
\frac{n(\text { relic })}{n(p)} \sim \frac{10^{-10}}{\alpha^{2}} \searrow 10^{-10} \quad \begin{array}{r}
\text { (strongly interacting) } \\
\text { (weally interacting) }
\end{array}
$$




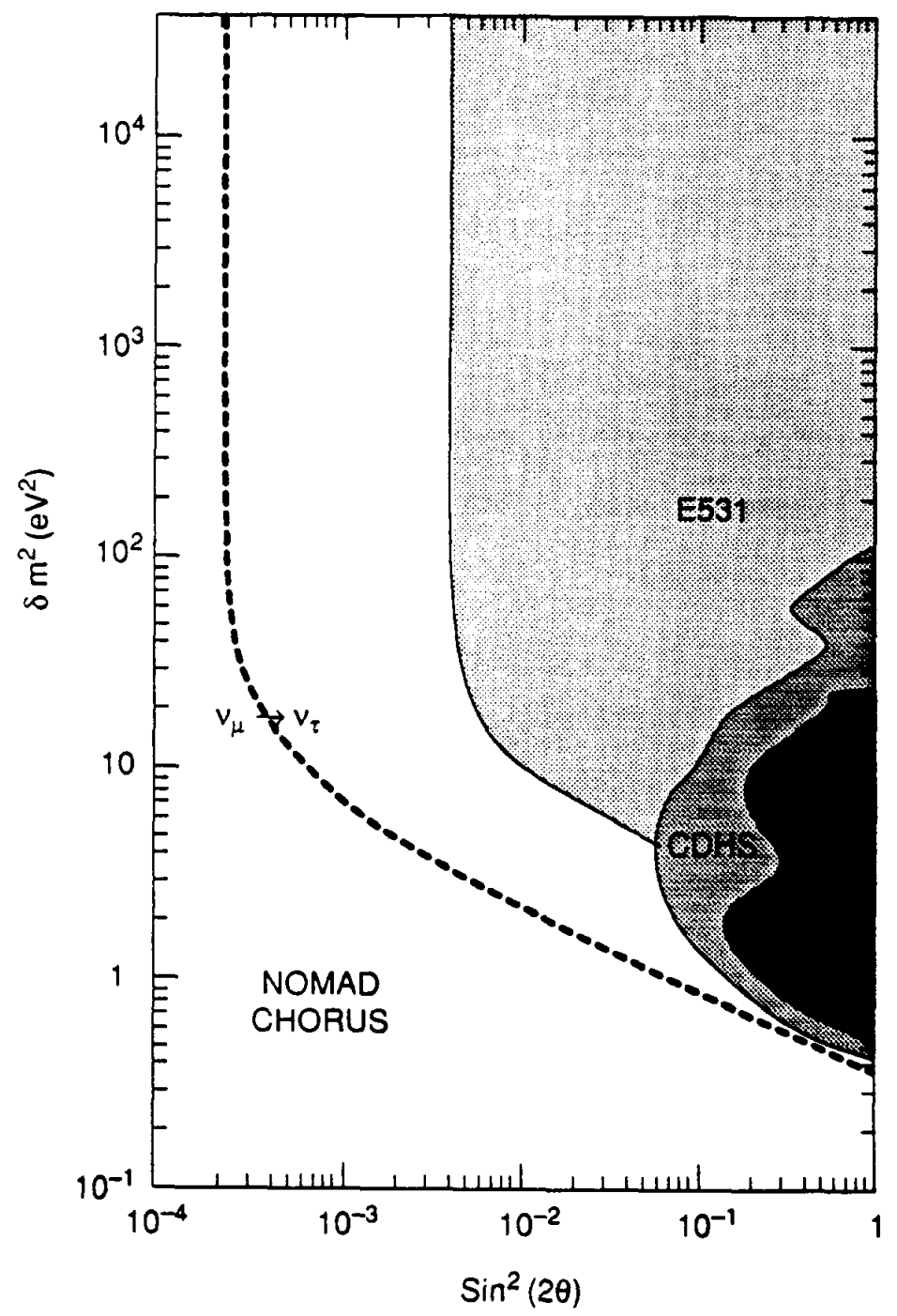

Fig. 5 - Present experimental limits on $\left(\nu_{\mu}, \nu_{r}\right)$ mixing in the $\left(\sin ^{2} \theta, \delta m^{2}\right)$ plane, and the planned sensitivity of the CHORUS and NOMAD experiments [27].

These estimates conflict with experimental upper limits [29] on the abundances of anomalous heavy isotopes relative to protons

$$
\frac{n \text { (relic) }}{n(p)} \lesssim 10^{-15} \text { to } 10^{-30} \text { for } 1 \text { GeV } \lesssim m_{\text {relic }} \lesssim 10 T e V
$$

Hence we conclude [10] that the stable relic particle must be electromagnetically neutral and only have weak interactions. 
Supersymmetric candidates include the sneutrinos $\dot{\nu}$ of spin zero, some mixture of $\dot{\gamma}, \dot{H}$ and $\check{Z}$, called a neutralino, of spin $1 / 2$. and the gravitino of spin $3 / 2$. The most plausible of these is the lightest neutralino. There are in the minimal supersymmetric extension of the Standard Model four neutralinos, whose masses and mixing are parametrized by three parameters: $m_{1 / 2}$, the umixed gaugino mass, $\mu$, a Higgs mixing parameter, and tan $\beta$, the rat io of supersymmetric Higgs vacuum expectation values. The composition of the lightest supersymmetric particle simplifies in the limits $m_{1 / 2} \rightarrow 0$, in which it is approximately a photino $\dot{\gamma}$, and $\mu \rightarrow 0$, in which limit it is a higgsino $\dot{H}$. However. experiments rule out either of these limits [30], and the generic lightest neutralino is a complicated mixture of $\dot{\gamma}, \dot{Z}$ and $\tilde{H}$. Constraints on neutralinos and their charged and strongly-interacting partners constrain the lightest supersymmetric particle

$$
m_{x} \gtrsim(10 \text { to } 20) \mathrm{GeV}
$$

As seen in Fig. 6, there are large regions of the neutralino parameter space where $\Omega_{\mathrm{LSP}}=$ $\rho_{\text {LSP }} / \rho_{\text {critical }} \simeq 1$ is possible. We therefore conclucle that the L.SP is a good candidate for the non-baryonic cold dark matter.
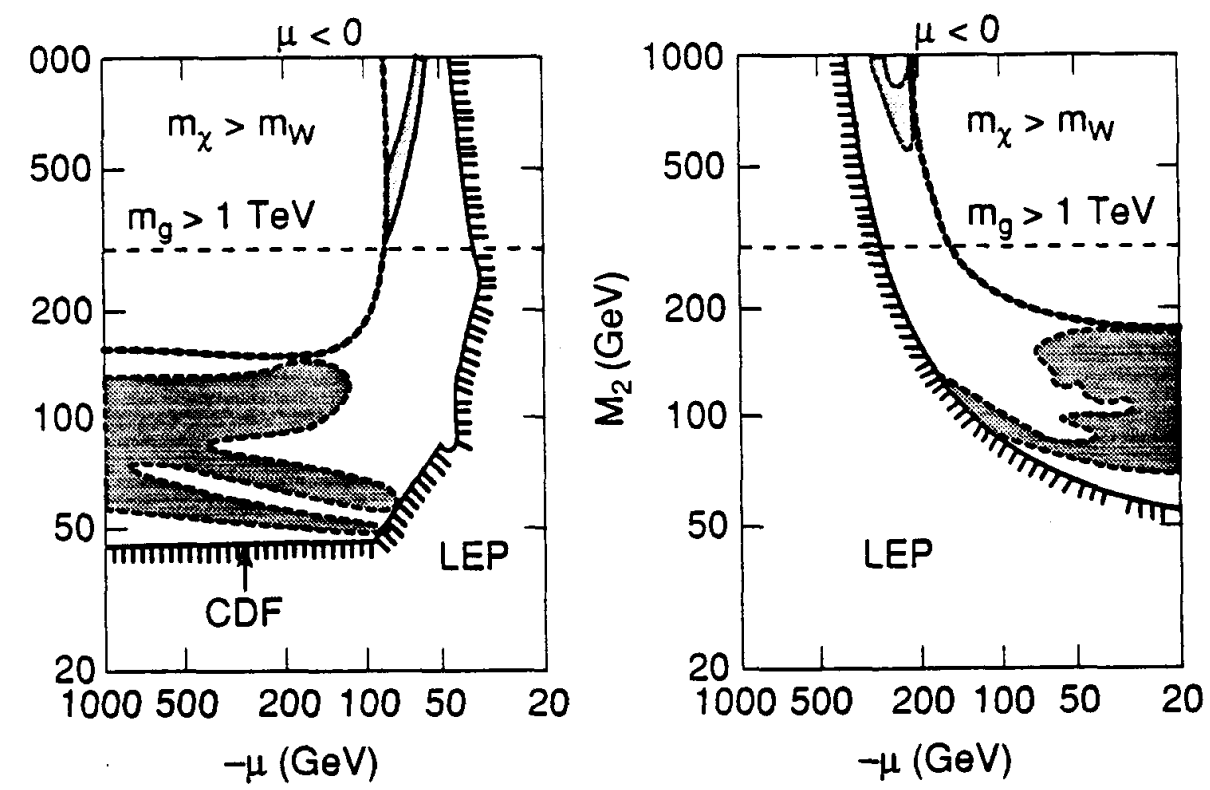

Fig. 6 - Regions of the $\left(\mu, m_{1 / 2}\right)$ plane excluded by LEP and CDF searches, together with regions where the lightest neutralino mass $m_{\Upsilon}<m_{W}$, and the relic neutralino density is in an "interesting" range $0.1<\Omega_{L S P} h^{2}<1$.

\section{Searches for Dark Matter Particles}

Some of the search strategies discussed in this section may also apply to other candidates for cold dark matter, but we will focus on neutralinos. 


\subsection{Annihilation in the Galactic Halo}

If the galactic halo is made of non-baryonic dark matter, then most of its density would consist of Cold Dark Matter particles. Every once in a while. these will find each other and annihilate in the halo, producing mainly $\bar{l}$ and $\bar{q} q$ final states, which eventually yield stable particles such as $\bar{p}, p, \epsilon^{+}, e^{-}, \gamma$ and $\nu$ in the cosmic rays [31]. Searches for antiprotons, positrons and $\gamma$ 's have been used [32] to set upper limits on the density of cold dark matter particles in the halo which are several times larger than the expected halo density. Since the fluxes are quadratically dependent on the relic density. and since the fluxes of antiprotons and positrons are uncertain because of uncertainties in the lifetime of cosmic ray particles within the galactic magnetic field, it is unlikely that this search strategy will be able to prove or disprove soon the hypothesis that the halo is made out of neutralinos.

\subsection{Annihilation in the Sun or Earth}

Here the idea [3:3] is that every once in a while a Cold Dark Matter particle will pass through the Sun and collide elastically with a nucleus inside it. This will cause it to lose recoil energy, which may transfer it from a hyperbolic orbit to an elliptic orbit with a perihelion below the solar surface. Repeated collisions with solar nuclei will then cause it to fall into an approximately isothermal distribution whose temperature is fixed by energy balance with the core of the Sun. ${ }^{1}$ The population of cold dark matter particles within the Sun is controlled by evaporation from the solar surface and/or annihilation in its core. The latter could give rise to an observable flux of high-energy solar neutrinos. which could be detected either directly in proton decay experiments or indirectly via the upward-going muons produced by their collisions in rock beneath the detectors.

It is important to note [34] that these high-energy solar neutrinos might also exhibit matterenhanced oscillations for values of the neutrino masses and mixing parameters that are consistent with experiment. These matter-enhanced neutrino oscillations could convert neutrinos produced inside the Sun into a species which is less detectable, such as the $\nu_{r}$. Therefore, experiments searching for high-energy solar neutrinos should always assume the worst, i.e., they should assume that the solar neutrinos have the flavour for which their detector has the smallest efficiency for detection. At the present time, assuming supersymmetric model parameters that give a relic density close to the critical density, and making other conservative assumptions, the upper limit on the local neutrino density is several times the expected halo density. In this case, there is reasonable hope that a new round of experiments will be able to explore the range of densities expected for Cold Dark Matter particles that constitute most of the galactic halo.

\subsection{Scattering in the Laboratory}

The most direct way to search for cold dark matter particles is via their elastic scattering on heavy nuclei in the laboratory [35]. The Cold Dark Matter particles are expected to have a

\footnotetext{
${ }^{1}$ The LSP is unable to cool the core of the Sun sufficient.ly to have an effect on the low-energy solar neutrino flux.
} 
typical velocity $v \simeq 300 \mathrm{~km} \mathrm{~s}^{-1}$, and to deposit a recoil energy

$$
\Delta E<m_{\imath} v^{2}=10\left(\frac{m_{\chi}}{10 \mathrm{GeV}}\right) \mathrm{keV}
$$

The effective interaction between neutralinos and quarks is given by

$$
\dot{L}_{e f f}=\sum_{q}\left[\left(\bar{\gamma} \gamma_{\mu} \gamma_{5} \chi\right)\left(\bar{q} \gamma^{n}\left(A_{q}\left(\frac{1-\gamma_{5}}{2}\right)+B_{q}\left(\frac{1+\gamma_{5}}{2}\right)\right) q\right)+\bar{\gamma} \chi C_{q} m_{q} \bar{q} q\right]
$$

The first term is due to $\tilde{f}$ and $Z$ exchange, and is spin-dependent. The second term is due to $H$ and $\tilde{f}$ mixing, and is spin-independent. The proton matrix element of the spin-dependent interaction may be written in the form

$$
M_{S D}(\backslash p \rightarrow \backslash p)=4 \mathrm{~s}_{\uparrow} \cdot \mathrm{s}_{p} \sum_{q \epsilon p}\left(B_{q}-A_{q}\right) \Delta q
$$

and similarly for the neutron. where the s are spin vectors. and the $\Delta q$ are the different quark contributions to the proton spin. In what follows, we will assume [36]

$$
\Delta n=0.77 \pm 0.08 . \Delta d=-0.49 \pm 0.08 . \Delta s=-0.15 \pm 0.08
$$

which comes from a fit to data on polarized $\mu p$ scattering, and is consistent with more recent data on polarized $\mu D,{ }^{3} \mathrm{He}$ scattering[37]. The proton matrix elements of the spin-independent interaction may be written as

$$
M_{S I}(x p \rightarrow \backslash p)=\left[\frac{\hat{f} m_{{ }_{u}} C_{u}+m_{d} C_{t}}{m_{u}+m_{d}}+f C_{s}+\frac{2}{27}(1-f-\hat{f})\left(C_{c}+C_{b}+C_{t}\right)\right]
$$

where the fractions of the proton mass carried by quarks are [36]

$$
\hat{f} \simeq 0.0 .5, f \simeq 0.2
$$

The spin-dependent interaction is dominant for light nuclei, whilst the spin-independent interaction is coherent and dominant for heavy nuclei.

Figure 7 displays figures of merit for spin-dependent LSP-nucleus scattering, as calculated [36] in a naive single-particle shell model, and as corrected by the more successful odd-group model [38]. We see that Fluorine is likely to be the best nucleus for monitoring the spindependent interaction. LSP-nucleus elastic scattering rates are given by

$$
R=\left(R_{S D}+R_{S I}\right) Y\left(\frac{4 m_{\Upsilon} m_{N}}{\left(m_{\mathrm{\Upsilon}}+m_{N}\right)^{2}}\right)\left(\frac{\rho_{\searrow}}{0.3 \mathrm{GeV} \mathrm{cm}^{-3}}\right)\left(\frac{v}{320 \mathrm{kms}^{-1}}\right) \text { events } k g^{-1} d^{-1}
$$

where $Y$ is the isotopic abundance, $\rho_{\mathrm{LSP}}$ is the local density of cold dark matter particles, and $v$ is the velocity of the LSP relative to the Earth. The quantity $R_{S D}$ is the spin-dependent interaction factor

$$
R_{S D}=5.5\left(\frac{10^{4} G_{i} V^{2}}{m_{0}^{2}-m_{\mathrm{x}}^{2}}\right) \lambda^{2} J(J+1) \zeta_{s}\left(\frac{m_{0}^{2}-m_{\chi}^{2}}{\epsilon^{2}} \sum_{q}\left(B_{q}-A_{q}\right) \Delta q\right)^{2}
$$




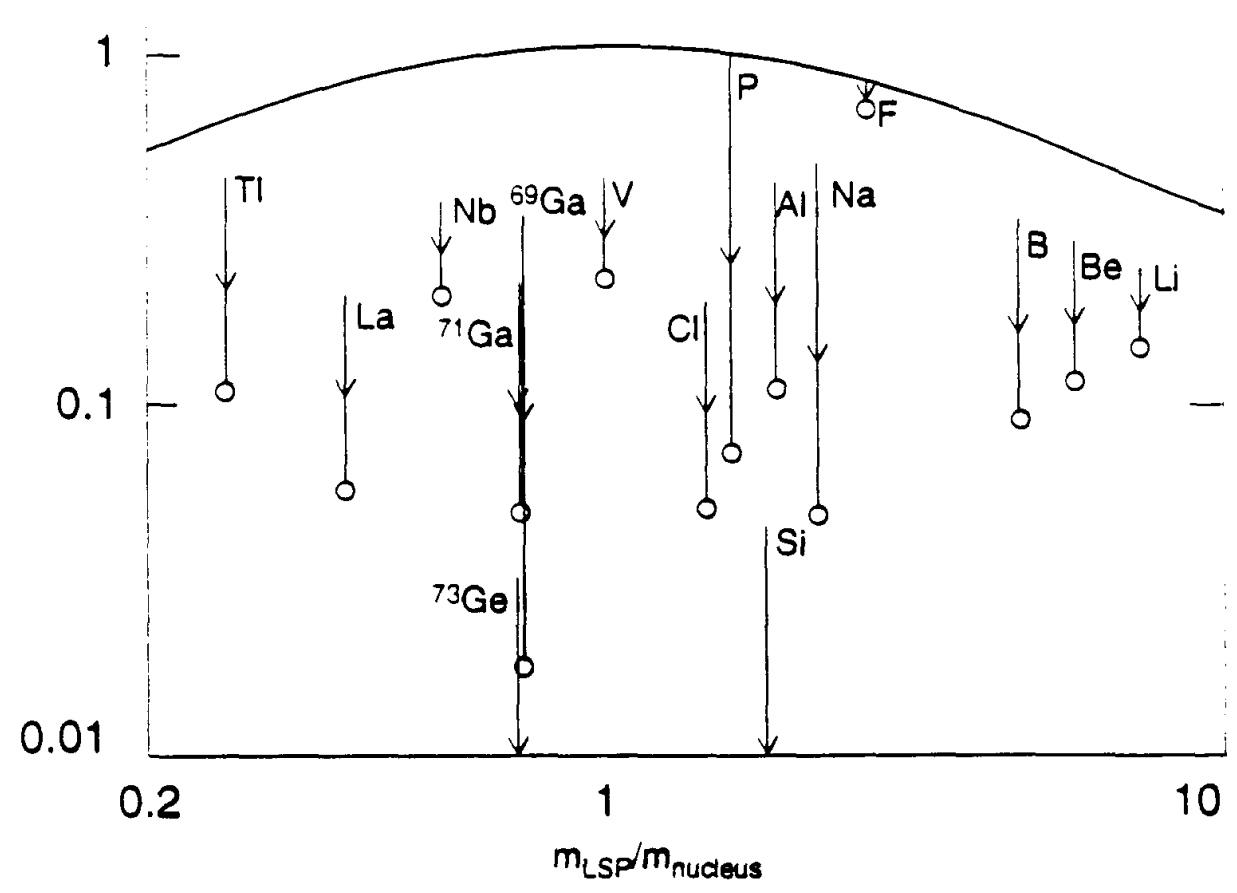

Fig. 7 - Figures of merit for spin-dependent neutralino-nucleus scattering. The tops of the arrows are the naive shell-model predictions, the bottoms are those found [36] in the odd-group model [38].

where $\lambda$ is a spin factor obtained using the odd-group model, $J$ is the nucleon spin, $\zeta_{s}$ is a spin form factor, and the $\Delta q$ were given in Eq. (36). The quantity $R_{S I}$ is the spin-independent interaction rate given by

$$
R_{S I}=210 m_{N}^{2} m_{\chi}^{4} \zeta_{c}\left(\hat{f} \frac{m_{u} C_{u}+m_{d} C_{d}}{m_{u}+m_{d}}+f C_{s}+\frac{2}{27}(1-f-\hat{f})\left(C_{c}+C_{b}+C_{t}\right)\right)^{2}
$$

where $\zeta_{c}$ is the charge form factor of the nucleus, and the $\hat{f}, f$ were given in Eq. (38). We adjust the supersymmetry model parameters so as to give critical density for the LSP for a present-day Hubble expansion rate $h_{0}=50 \mathrm{~km} / \mathrm{second} / \mathrm{megaparsec}$. The resulting elastic scattering rates depend on the masses of the supersymmetric Higgs particles, as seen in Fig. 8. We see there that Fluorine and Germanium are useful in complementary regions of the LSP parameter space, whilst Thallium may be useful in regions similar to those of Germanium. Figure 9 shows in the case of Germanium the "probability" of detection, defined to be the fraction of the parameter space where $\Omega_{r m L S P}=1$ is possible, in which the elastic scattering rate is "observable", in the sense of being above 0.1 events per kilogram per day [36]. We see that there is quite a good probability of detection, although this cannot be guaranteed. We also see that the regions of supersymmetric parameter space that are detectable in cold dark matter searches complement and extend those accessible to LEP II and the LHC. 


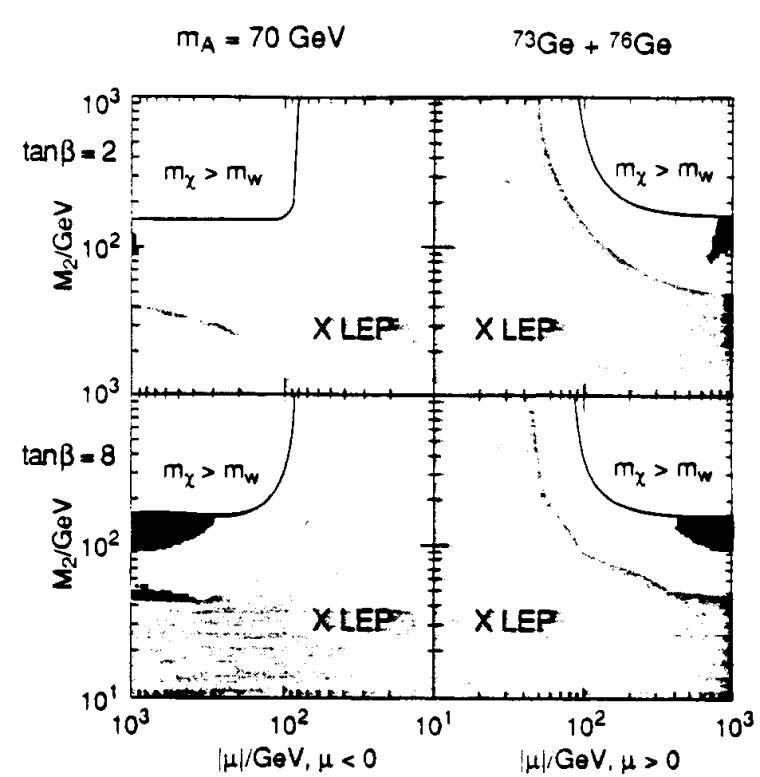

(a)

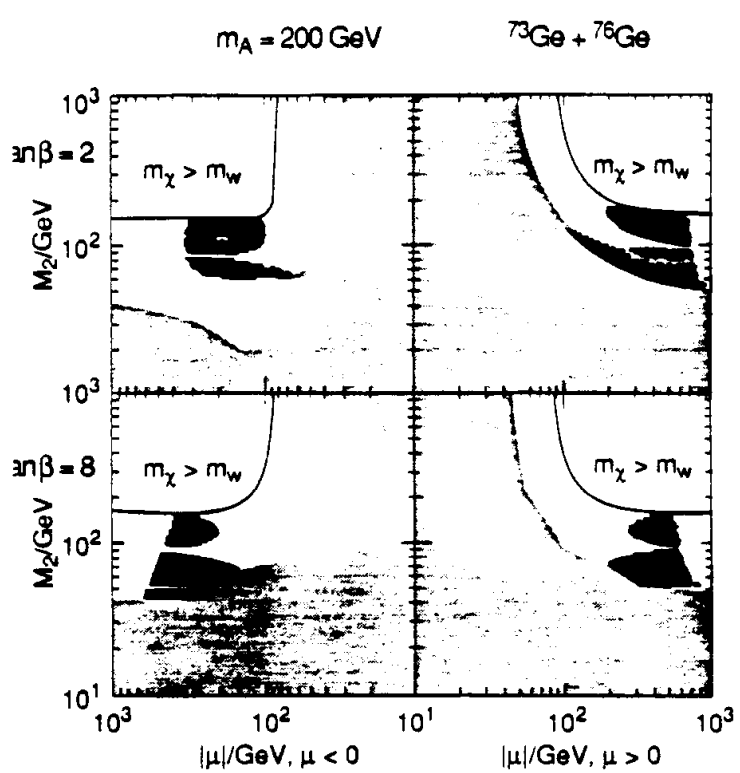

(b)

Fig. 8 - Elastic scattering rates for (a), (b) ${ }^{73} \mathrm{Ge}+{ }^{76} \mathrm{Ge}$, for the pseudoscalar Higgs mass $m_{A}=70 \mathrm{GeV}$ in (a), $200 \mathrm{GeV}$ in (b) [36]. The rates are above 0.1 events $k g^{-1} d^{-1}$ in the shaded regions.
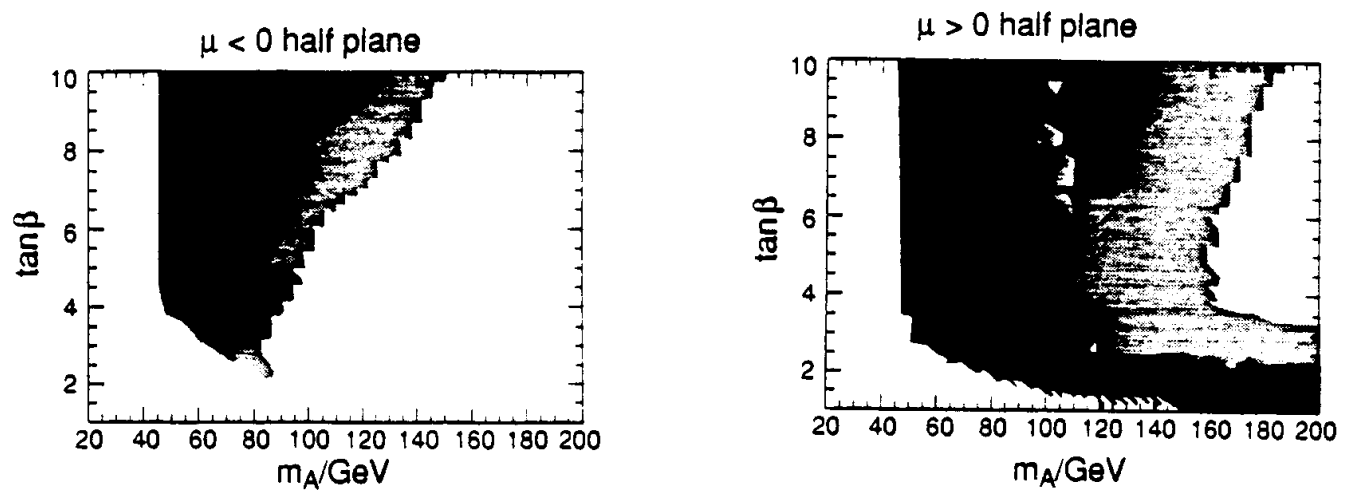

Fig. 9 - The "probability" of detecting neutralino dark matter, as defined by the fraction of the parameter space where the critical density $\Omega_{L S P}=1$ can be attained in which the $\chi-G e$ elastic scattering rate exceeds 0.1 events $\mathrm{kg}^{-1} \mathrm{~d}^{-1}[36]$. 


\section{The Copernican Challenge}

Copernicus showed that we do not live at the centre of the Universe. Modern astrophysicists claim that we are not even made of the same stuff as most of the matter in the Universe. The challenge to our experimental colleagues is to prove this assertion!

\section{References}

[1] W. Fowler and F. Hoyle, Ap.J.Supp. 9 (1964) 201;

R. Wagoner, Ap.J. 179 (1973) 34:3.

J. Yang, M.S. Turner, G. Steignan, D.N. Schramm and K.A. Olive, Ap.J. 281 (1984) 493.

B. Pagel, Physica Scripta T36 (1991) 7.

[2] J.H. Applegate and C.J. Hogan. Phys.Rev. D31 (1985) 30:37;

J.H. Applegate, C..J. Hogan and R. Scherrer. Phys.Rev. D35 (198i) 1151;

C. Alcock. G.M. Fuller and G.J. Mathews, Ap.J. 320 (1987) 439.

[3] J. Ignatios, K. Kajantie, H. Kurki-Sainio and M. Laine, Helsinki Preprint HU-TFT-93-43 (1993).

[4] See the parallel session on Heavy Ion Collisions at the Conference.

[5] D. Thomas, D.N. Schramm. K.A. Olive and B.D. Fields, Ap.J. 406 (1993) 569.

[6] F. Dydak, Parallel session talk;

G. Altarelli, CERN Preprint TH. 7072/93 (1993)

[7] J. Ellis, G.L. Fogli and E. Lisi. CERN Preprint TH. 6967/93 (1993).

[8] J. Iliopoulos, Parallel session talk.

[9] S. Ferrara, Parallel session talk;

P. Fayet and S. Ferrara. Physics Reports 32 (1977) 251.

[10] H. Goldberg, Phys. Rev. Lett. 50 (1983) 1419;

J. Ellis, J.S. Hagelin, D.V. Nanopoulos, K.A. Olive and M. Srednicki, Nucl. Phys. B238 (1984) 453.

[11] P. Langacker, University of Pennsylvania preprint UPR 0238T (1990);

J. Ellis, S. Kelley and D.V. Nanopoulos, Phys. Lett. B249 (1990) 441:

U. Amaldi, W. de Boer and H. Furstenau, Phys. Lett. B260 (1991) 447.

[12] A.A. Starobinsky, Phys. Lett. 91B (1980) 99;

A. Guth, Phys. Rev. D23 (1981) 349.

[13] G.F. Smoot et al., Ap. J. Lett. 396 (1992) Ll;

E.L.Wright et al., Ap. J. Lett. 396 (1992) L13. 
[14] A. Guth and E. Weinberg, Phys. Rev. D23 (1981) 876;

S.W. Hawking, I.G. Moss and J.M. Stewart, Phys. Rev. D26 (1982) 2681.

[15] A.D. Linde, Phys. Lett. 108B (1982) 389;

A. Albrecht and P.J. Steinhardt, Phys. Rev. Lett. 48 (1982) 1220.

[16] E.J. Copeland. E.IV. Kolb, A.R. Liddle and J.E. Lidsey, Sussex preprints SUSSEX-AST 93/3, 4-1 (1993):

J.R. Bond et al., CITA/University of Pennsylvania/Oxford preprint (1993).

[17] J. Bardeen, P.J. Steinhardt and M.S. Turner, Phys. Rev. D28 (1983) 679;

A.H. Guth and S.-Y. Pi, Phys. Rev. Lett. 49 (1982) 1110;

A.A. Starobinsky, Phys. Lett. 117B (1982) 175;

S.W. Hawking, Phys. Lett. 115B (1982) 295.

[18] D. Hegyi and K.A. Olive, Astrophys. J. 303 (1986) 56.

[19] C. Alcock et al., Valure 365 (199:3) 621;

E. Aubourg et al., Nature 365 (1993) 6233.

[20] See, for example:

G. Efstathiou, J.R. Bond and S.D.M. White, Mon. Not. R. Ast. Soc. 258 (1992) 1P;

M.S. Turner, FNAL preprint Conf-92/313-A (1992).

[21] G. Efstathiou, private communication (1993).

[22] B.W. Lee and S. Weinberg, Phys. Rev. Lett. 39 (1977) 165;

P. Hut, Phys. Lett. 69B (1977) 85.

[23] S. Dimopoulos, Phys. Lett. B246 (1990) 347.

[24] P. Anselmann et al., Phys. Lett. B285 (1982) 390, and references therein.

[25] T. Yanagida, Proc. Workshop on the Unified Theory and the Baryon Number in the Universe (KEK, Japan, 1979);

R. Slansky, talk at the Sanibel Symposium, Caltech preprint CALT-68-709 (1979).

[26] J. Ellis, J. Lopez and D.V. Nanopoulos, Phys. Lett. B292 (1992) 189.

[27] CHORUS Collaboration, N. Armenise et al., CERN-SPSC/90-42 (1990);

NOMAD Collaboration. P. Astier et al., CERN-SPSC/91-21 (1991).

[28] C. Rubbia and J.P. Revol, private communications (1993).

[29] J. Rich, M. Spiro and J. Lloyd-Owen, Physics Reports 151 (1987) 239.

[30] J. Ellis, G. Ridolfi and F. Zwirner, Phys. Lett. B237 (1990) 423.

[31] J. Silk and M. Srednicki, Phys. Rev. Lett. 53 (1984) 624. 
[32] J. Ellis et al., Phys. Lett. B214 (1988) 403.

[33] J. Silk, K.A. Olive and M. Srednicki, Nucl. Phys. B279 (1987) 804.

[34] J. Ellis, R.A. Flores and S. Masood, Nucl. Phys. B294 (1992) 229.

[35] M. Goodman and E. Witten, Phys. Rev. D30 (1985) 3059.

[36] J. Ellis and R.A. Flores, Phys. Lett. B263 (1991) 259; Nucl. Phys. B400 (1993) 25;

J. Ellis and R.A. Flores, Nucl. Phys. B307 (1988) 883.

[37] J. Ellis and M. Karliner, Phys. Lett. B313 (1993) 131.

[38] J. Engel and P. Vogel, Phys. Rev. D40 (1989) 3132. 